\title{
ALGEBRAIC COHOMOLOGY OF TOPOLOGICAL GROUPS
}

\author{
BY DAVID WIGNER
}

\author{
Communicated by David Buchsbaum, December 24, 1969
}

In this note we discuss algebraic cohomology groups of topological groups. Eilenberg-Maclane [3] and Hopf [6] introduced the notion of algebraic cohomology of abstract groups, and definitions taking into account the topology are given in [2], [4], [5], and [8]. We give a definition which coincides with the usual one for discrete groups and generalizes those in [2], [5] and [8] for topological groups. It has the good functorial properties of being an "exact connected sequence of functors" in a suitable sense, and of being effaceable and universal.

All groups and modules considered will be Hausdorff.

The classical theory assigns to an abstract group $G$ and a $G$ module $A$ an exact connected sequence of functors $H^{i}(G, A)$ $(0 \leqq i<\infty)$. It can be shown that this sequence is universal and effaceable, and for any other effaceable exact connected sequence of functors $\check{H}$ with $\check{H}^{0}(G, A) \cong H^{0}(G, A)$ for all $A$, one has $\check{H}^{i}(G, A)$ $\cong H^{i}(G, A)$ for all $i$ by Buchsbaum's criterion.

If $G$ is a topological group, a topological $G$-module $A$ will mean an abelian topological group $A$ with a jointly continuous action of $G$ satisfying $g\left(a+a^{\prime}\right)=g a+g a^{\prime},\left(g g^{\prime}\right) a=g\left(g^{\prime} a\right), l a=a$. We show that topological $G$-modules form a quasi-abelian category in the sense of Yoneda [9], and define $H^{i}(G, A)=\operatorname{Ext}^{i}(Z, A)$ where Ext is given by the definition of Yoneda for the quasi-abelian category of topological $G$-modules. $H^{0}(G, A)$ will then be the abstract group of points of $A$ fixed under the action of $G$. If the underlying spaces of all groups and modules in question are limits of sequences of compact sets, we show that $H^{1}$ and $H^{2}$ have the obvious interpretations in terms of continuous crossed homomorphisms and extensions of topological groups.

Yoneda shows that with the appropriate definitions the Ext ${ }^{i}$ form an effaceable exact connected sequence of functors; one can further show that they are universal and prove a modified form of Buchsbaum's criterion. The "bar construction" semisimplicial resolution of an abstract group $G$ [7] becomes a semisimplicial space in an obvious way if $G$ is a topological group. If $A$ is a $G$-module consider the sheaf of germs of $A$-valued functions on each space of this semisimplicial resolution. The canonical resolutions of these sheaves give rise to a

AMS Subject Classifications. Primary 2210.

Key Words and Phrases. Cohomology group, Lie algebra, classifying space of a topological group. 
double complex. $\hat{H}^{i}(G, A)$ will denote the $i$ th cohomology group of this double complex. We then have:

THEOREM. If $G$ is locally compact, $\sigma$-compact and finite dimensional or a countable $\mathrm{CW}$-complex and $A$ is complete metric and either locally connected or locally compact, then $H^{i}(G, A)=\hat{H}^{i}(G, A)$.

Theorem. Suppose $G$ is as in the previous theorem. If $A$ is discrete, $H^{i}(G, A)$ is isomorphic to the cohomology $H^{i}\left(B_{G}, A\right)$ of the classifying space $B_{G}$ with coefficients in $A$.

THEOREM. If $G$ is a connected Lie group, and $A$ a finite dimensional vector space which is a differentiable G-module, then $H^{i}(G, A) \cong H_{\mathrm{Lie}}^{i}(\mathrm{G}, \mathfrak{K}, A)$; the latter is the cohomology of the Lie algebra $S$ of $G$ modulo the Lie algebra $\nVdash K$ of a maximal compact subgroup.

\section{REFERENCES}

1. D. A. Buchsbaum, Satellites and universal functors, Ann. of Math. (2) 71 (1960), 199-209. MR $22 \# 3751$.

2. A. Douady, "Cohomologie des groupes compacts totalement discontinus," Seminaire Bourbaki, 1959/60, exposé 189, Secrétariat mathematique, Paris, 1960. MR 23 \#A2273.

3. S. Eilenberg and S. Maclane, Relations between homology and homotopy groups, Proc. Nat. Acad. Sci. U.S.A. 29 (1943), 155-158. MR 4, 224; MR 5, 328.

4. I. M. Gel'fand and D. B. Fuks, Topology of noncompact Lie groups, Funkcional. Anal. i Priložen. 1 (1967), no. 4, 33-45 = Functional Anal. Appl. 1 (1967), 285-295. MR 37 \#2253.

5. G. P. Hochschild and G. D. Mostow, Cohomology of Lie groups, Illinois J. Math. 6 (1962), 367-401. MR $26 \# 5092$.

6. H. Hopf, Über die Bettischen Gruppen, die zu einer beliebigen Gruppe gehören, Comment. Math. Helv. 17 (1945), 39-79. MR 6, 279.

7. S. MacLane, Homology, Die Grundlehren der math. Wissenschaften, Band 114, Academic Press, New York and Springer-Verlag, Berlin and New York, 1963. MR 28 \#122.

8. C. C. Moore, Extensions and low dimensional cohomology theory of locally compact groups. I, II, Trans. Amer. Math. Soc. 113 (1964), 40-86. MR 30 \#2106.

9. N. Yoneda, On Ext and exact sequences, J. Fac. Sci. Univ. Tokyo Sect. I 8 (1960), 507-576. MR 37 \#1445.

Stanford University, Stanford, California 94305 